\title{
EXISTENCE AND UNIQUENESS OF SOLUTIONS OF STOCHASTIC DIFFERENTIAL EQUATIONS WITH NON-LIPSCHITZ DIFFUSION AND POISSON MEASURE
}

UDC 519.21

\author{
V. P. ZUBCHENKO AND YU. S. MISHURA
}

\begin{abstract}
The existence and uniqueness of a solution of a stochastic differential equation with a non-Lipschitz diffusion for cases of both centered and non-centered Poisson measures is proved. We prove that the pathwise uniqueness of a solution and the existence of a weak solution imply the existence of a strong solution for such equations.
\end{abstract}

\section{INTRODUCTION}

Many papers and monographs are devoted to studies of the existence and uniqueness of solutions of stochastic differential equations. In particular, these problems are considered in the books [1] and 2] for stochastic equations with Lipschitz coefficients and with a jump component or for equations with non-Lipschitz (Hölder) diffusion but without a jump component. Some results concerning the existence and uniqueness of weak solutions of diffusion type equations are obtained in [3]. Equations of this type with non-Lipschitz coefficients are considered in 44. It is proved in [5] that the pathwise uniqueness and existence of a weak solution of diffusion type equations imply the existence of a strong solution.

In the current paper, we consider the following stochastic differential equation:

$$
\begin{aligned}
d X(t)= & a(X(t)) d t+g(X(t)) d W(t) \\
& +\int_{\mathbb{R}} q_{1}(X(t), y) \tilde{\nu}(d t, d y)+\int_{\mathbb{R}} q_{2}(X(t), y) \mu(d t, d y),
\end{aligned}
$$

where $W(t)$ is a Wiener process, $\nu(d t, d y)$ a Poisson measure such that

$$
\mathrm{E} \nu(d t, d y)=\Pi(d y) d t
$$

$\tilde{\nu}(d t, d y)=\nu(d t, d y)-\Pi(d y) d t$ a centered Poisson measure, $\Pi(\cdot)$ a $\sigma$-finite measure in the $\sigma$-algebra of Borel sets of $\mathbb{R}, \mu(d t, d y)$ a noncentered Poisson measure such that $\mathrm{E} \mu(d t, d y)=m(d y) d t$, and $m(\cdot)$ a finite measure in the $\sigma$-algebra of Borel sets of $\mathbb{R}$. Let $(\Omega, \mathcal{F}, \mathrm{P})$ be a probability space equipped with a flow of $\sigma$-algebras $\mathcal{F}_{t}$ and completed with all null random events of the $\sigma$-algebra $\mathcal{F}_{0}$. The Wiener process $W(t)$, centered Poisson measure $\tilde{\nu}(d t, d y)$, and noncentered Poisson measure $\mu(d t, d y)$ are adapted to the flow $\mathcal{F}_{t}$ and are jointly independent; the random variable $X(0)$ is $\mathcal{F}_{0}$-measurable. The coefficients

2000 Mathematics Subject Classification. Primary 60H10; Secondary 60H05, 60J65.

Key words and phrases. Stochastic differential equation, non-Lipschitz diffusion, Poisson measure, weak solution, existence and uniqueness of solution.

The authors are grateful to the European Commission for support of their investigations in the framework of the Program "Marie Curie Actions", grant "Multifractionality" PIRSES-GA-2008-230804. 
$a(x), g(x), q_{1}(x, y)$, and $q_{2}(x, y)$ are nonrandom measurable functions. The drift coefficient $a(x)$ is Lipschitzian, while the diffusion $g(x)$ is Hölderian (the precise assumptions imposed on the diffusion coefficient are listed in the statement of Theorem 2.1).

We show that the pathwise uniqueness and existence of a weak solution imply the existence of a strong solution for equation (1) and we prove the existence and uniqueness of a strong solution for this equation.

\section{Pathwise uniqueness}

Along with equation (11) we consider the following stochastic differential equation:

$$
d X(t)=a(X(t)) d t+g(X(t)) d W(t)+\int_{\mathbb{R}} q_{1}(X(t), y) \tilde{\nu}(d t, d y) .
$$

Theorem 2.1. I. Let the coefficients of equation (2) be such that

1) the functions $a(x), g(x)$, and $\int_{\mathbb{R}}\left|q_{1}(x, y)\right|^{2} \Pi(d y)$ are bounded;

$2)$ there exists an increasing function $\rho(u)$ defined on the interval $[0, \infty)$ and such that $\rho(0)=0$,

$$
\int_{0+} \rho^{-2}(u) d u=\infty
$$

and $|g(x)-g(y)| \leq \rho(|x-y|)$ for all $x, y \in \mathbb{R}$

3) $\left|a\left(x_{1}\right)-a\left(x_{2}\right)\right| \leq k\left|x_{1}-x_{2}\right|$,

$$
\int_{\mathbb{R}}\left|q_{1}\left(x_{1}, y\right)-q_{1}\left(x_{2}, y\right)\right| \Pi(d y) \leq k\left|x_{1}-x_{2}\right|
$$

for all $x_{1}, x_{2} \in \mathbb{R}$, where $k$ is a certain constant.

Then the solution of equation (2) is pathwise unique.

II. Let the above conditions 1)-3) hold for the coefficients of equation (1) and let the function $q_{2}(x, y)$ be continuous with respect to $x$ in measure $m(d y)$. Then the solution of equation (11) is unique.

Remark 2.1. Assertion I of Theorem 2.1 is proved in [6] for $q_{2}=0$ under a non-Lipschitz condition imposed on $\int_{\mathbb{R}}\left|q_{1}(x, y)\right| \Pi(d y)$. Assertion II is obtained in [2] for the case of Lipschitzian drift and diffusion coefficients. The method of the proof of the corresponding results below is similar to that used in [6] and [2].

Proof. First we prove assertion I of the theorem. Let the sequence of real numbers

$$
1>a_{1}>a_{2}>\cdots>a_{n}>\cdots>0
$$

be defined by the following conditions:

$$
\int_{a_{1}}^{1} \rho^{-2}(u) d u=1, \quad \int_{a_{2}}^{a_{1}} \rho^{-2}(u) d u=2, \quad \ldots, \quad \int_{a_{n}}^{a_{n-1}} \rho^{-2}(u) d u=n, \quad \ldots
$$

Obviously $a_{n} \rightarrow 0$ as $n \rightarrow \infty$. Let $\psi_{n}(u), n=1,2, \ldots$, be a continuous function such that its support belongs to $\left(a_{n}, a_{n-1}\right), 0 \leq \psi_{n}(u) \leq 2 \rho^{-2}(u) / n$, and $\int_{a_{n}}^{a_{n-1}} \psi_{n}(u) d u=1$. Clearly such a function exists. Put

$$
\varphi_{n}(x)=\int_{0}^{|x|} d y \int_{0}^{y} \psi_{n}(u) d u, \quad x \in \mathbb{R} .
$$

Then $\varphi_{n} \in C^{2}(\mathbb{R}),\left|\varphi_{n}^{\prime}(x)\right| \leq 1$, and $\varphi_{n}(x) \uparrow|x|$ as $n \rightarrow \infty$.

Let

$$
\left(X_{1}(t), W(t), \tilde{\nu}(d t, d y)\right) \quad \text { and } \quad\left(X_{2}(t), W(t), \tilde{\nu}(d t, d y)\right)
$$


be two solutions of equation (2) defined on the same probability space with the same flows and such that $X_{1}(0)=X_{2}(0)=X$. Then

$$
\begin{aligned}
X_{1}(t)-X_{2}(t)= & \int_{0}^{t}\left[a\left(X_{1}(s)\right)-a\left(X_{2}(s)\right)\right] d s+\int_{0}^{t}\left[g\left(X_{1}(s)\right)-g\left(X_{2}(s)\right)\right] d W(s) \\
& +\int_{0}^{t} \int_{\mathbb{R}}\left[q_{1}\left(X_{1}(s), y\right)-q_{1}\left(X_{2}(s), y\right)\right] \tilde{\nu}(d s, d y) .
\end{aligned}
$$

Here and in what follows we consider separable versions of stochastically equivalent processes.

Since the coefficients of the equation are bounded, E $\left|X_{1}(t)-X_{2}(t)\right|<\infty$. Applying the Itô formula to the function $\varphi_{n}(x)$ we obtain

$$
\begin{aligned}
\varphi_{n}\left(X_{1}(t)-X_{2}(t)\right) \\
=\int_{0}^{t} \varphi_{n}^{\prime}\left(X_{1}(s)-X_{2}(s)\right)\left[g\left(X_{1}(s)\right)-g\left(X_{2}(s)\right)\right] d W(s) \\
\quad+\int_{0}^{t} \varphi_{n}^{\prime}\left(X_{1}(s)-X_{2}(s)\right)\left[a\left(X_{1}(s)\right)-a\left(X_{2}(s)\right)\right] d s \\
\quad+\frac{1}{2} \int_{0}^{t} \varphi_{n}^{\prime \prime}\left(X_{1}(s)-X_{2}(s)\right)\left[g\left(X_{1}(s)\right)-g\left(X_{2}(s)\right)\right]^{2} d s \\
+\int_{0}^{t} \int_{\mathbb{R}}\left[\varphi_{n}\left(X_{1}(s)-X_{2}(s)+q_{1}\left(X_{1}(s), y\right)-q_{1}\left(X_{2}(s), y\right)\right)\right. \\
\quad-\varphi_{n}\left(X_{1}(s)-X_{2}(s)\right) \\
\left.\quad-\left(q_{1}\left(X_{1}(s), y\right)-q_{1}\left(X_{2}(s), y\right)\right) \varphi_{n}^{\prime}\left(X_{1}(s)-X_{2}(s)\right)\right] \Pi(d y) d s \\
\quad+\int_{0}^{t}\left[\varphi_{\mathbb{R}}\left[\varphi_{n}\left(X_{1}(s)-X_{2}(s)+q_{1}\left(X_{1}(s), y\right)-q_{1}\left(X_{2}(s), y\right)\right)\right] \tilde{\nu}(d s, d y) .\right.
\end{aligned}
$$

The expectations of the first and last terms on the right hand side of the latter equality are equal to zero. We show this, for example, for the last term. Indeed, applying the Lagrange formula to $\varphi_{n} \in C^{2}(\mathbb{R})$ we get

$$
\begin{aligned}
& \varphi_{n}\left(X_{1}(s)-X_{2}(s)+q_{1}\left(X_{1}(s), y\right)-q_{1}\left(X_{2}(s), y\right)\right)-\varphi_{n}\left(X_{1}(s)-X_{2}(s)\right) \\
& \quad=\varphi_{n}^{\prime}(\theta)\left(q_{1}\left(X_{1}(s), y\right)-q_{1}\left(X_{2}(s), y\right)\right)
\end{aligned}
$$

where $\theta=\theta(n, y)$ is a certain intermediate point. Since $\left|\varphi_{n}^{\prime}(\theta)\right| \leq 1$, we have

$$
\begin{aligned}
& \mathrm{E} \int_{0}^{t} \int_{\mathbb{R}} \mid \varphi_{n}\left(X_{1}(s)-X_{2}(s)+q_{1}\left(X_{1}(s), y\right)\right.\left.-q_{1}\left(X_{2}(s), y\right)\right) \\
&-\left.\varphi_{n}\left(X_{1}(s)-X_{2}(s)\right)\right|^{2} \Pi(d y) d s \\
& \leq \mathrm{E} \int_{0}^{t} \int_{\mathbb{R}}\left|\varphi_{n}^{\prime}(\theta)\left(q_{1}\left(X_{1}(s), y\right)-q_{1}\left(X_{2}(s), y\right)\right)\right|^{2} \Pi(d y) d s \\
& \leq \mathrm{E} \int_{0}^{t} \int_{\mathbb{R}}\left|q_{1}\left(X_{1}(s), y\right)\right|^{2} \Pi(d y) d s+\mathrm{E} \int_{0}^{t} \int_{\mathbb{R}}\left|q_{1}\left(X_{2}(s), y\right)\right|^{2} \Pi(d y) d s<\infty .
\end{aligned}
$$

Taking into account the martingale properties of stochastic integrals, we see that the expectation of the last term of equation (3) is equal to zero, indeed. The same method 
proves this statement for the first term, too. Thus

$$
\begin{aligned}
& \mathrm{E}\left[\varphi_{n}\left(X_{1}(t)-X_{2}(t)\right)\right] \\
& =\mathrm{E}\left[\int_{0}^{t} \varphi_{n}^{\prime}\left(X_{1}(s)-X_{2}(s)\right)\left\{a\left(X_{1}(s)\right)-a\left(X_{2}(s)\right)\right\} d s\right] \\
& +\frac{1}{2} \mathrm{E}\left[\int_{0}^{t} \varphi_{n}^{\prime \prime}\left(X_{1}(s)-X_{2}(s)\right)\left\{g\left(X_{1}(s)\right)-g\left(X_{2}(s)\right)\right\}^{2} d s\right] \\
& +\mathrm{E}\left[\int _ { 0 } ^ { t } \int _ { \mathbb { R } } \left\{\varphi_{n}\left(X_{1}(s)-X_{2}(s)+q_{1}\left(X_{1}(s), y\right)-q_{1}\left(X_{2}(s), y\right)\right)\right.\right. \\
& \quad-\varphi_{n}\left(X_{1}(s)-X_{2}(s)\right) \\
& \left.\left.\quad-\left(q_{1}\left(X_{1}(s), y\right)-q_{1}\left(X_{2}(s), y\right)\right) \cdot \varphi_{n}^{\prime}\left(X_{1}(s)-X_{2}(s)\right)\right\} \Pi(d y) d s\right] \\
& =I_{1}+I_{2}+I_{3},
\end{aligned}
$$

where

$$
\begin{gathered}
\left|I_{1}\right| \leq \int_{0}^{t} \mathrm{E}\left[\left|a\left(X_{1}(s)\right)-a\left(X_{2}(s)\right)\right|\right] d s \leq \int_{0}^{t} k\left[\mathrm{E}\left(\left|X_{1}(s)-X_{2}(s)\right|\right)\right] d s, \\
\left|I_{2}\right| \leq \frac{1}{2} \int_{0}^{t} \mathrm{E}\left[\frac{2}{n} \rho^{-2}\left(\left|X_{1}(s)-X_{2}(s)\right|\right) \rho^{2}\left(\left|X_{1}(s)-X_{2}(s)\right|\right)\right] d s \leq \frac{t}{n} \rightarrow 0, \quad n \rightarrow \infty .
\end{gathered}
$$

Consider the term $I_{3}$. Applying the Lagrange formula to $\varphi_{n} \in C^{2}(\mathbb{R})$, we get

$$
\begin{aligned}
\left|I_{3}\right| \leq \mathrm{E} \int_{0}^{t} \int_{\mathbb{R}} \mid \varphi_{n}^{\prime}(\theta)\left(q_{1}\left(X_{1}(s), y\right)-q_{1}\left(X_{2}(s), y\right)\right) \\
\quad-\varphi_{n}^{\prime}\left(X_{1}(s)-X_{2}(s)\right)\left(q_{1}\left(X_{1}(s), y\right)-q_{1}\left(X_{2}(s), y\right)\right) \mid \Pi(d y) d s \\
\leq 2 \mathrm{E} \int_{0}^{t} \int_{\mathbb{R}}\left|q_{1}\left(X_{1}(s), y\right)-q_{1}\left(X_{2}(s), y\right)\right| \Pi(d y) d s \leq \int_{0}^{t} k\left[\mathrm{E}\left(\left|X_{1}(s)-X_{2}(s)\right|\right)\right] d s .
\end{aligned}
$$

Passing to the limit as $n \rightarrow \infty$ yields

$$
\mathrm{E}\left(\left|X_{1}(t)-X_{2}(t)\right|\right) \leq \int_{0}^{t} k\left[\mathrm{E}\left(\left|X_{1}(s)-X_{2}(s)\right|\right)\right] d s .
$$

This implies that

$$
\mathrm{E}\left(\left|X_{1}(s)-X_{2}(s)\right|\right)=0 .
$$

Since this process is right continuous, $X_{1}(t)=X_{2}(t)$ almost surely. Therefore the first statement of Theorem 2.1 is proved.

Now we turn to the proof of the second statement of Theorem 2.1. Consider equation (11). Let $\tau_{1}<\tau_{2}<\cdots$ be sequential jumps of the Poisson process $\mu([0, t], \mathbb{R})$. Since the process $\mu$ is homogeneous with parameter $m(\mathbb{R})$, only a finite number of jumps occur in every finite interval. Let $\tau_{0}=0$. It is sufficient to prove the uniqueness of a solution of equation (11) in the interval $\left[\tau_{k}, \tau_{k+1}\right]$ if $X\left(\tau_{k}\right)$ is given. Then the induction shows that a solution of equation (11) is unique in an arbitrary interval $\left[0, \tau_{k}\right]$ and $\bigcup\left[0, \tau_{k}\right]=\mathbb{R}_{+}$.

Let $X_{1}(t)$ and $X_{2}(t)$ be two solutions of equation (1) in the interval $\left[\tau_{k}, \tau_{k+1}\right.$ [such that $X_{1}\left(\tau_{k}\right)=X_{2}\left(\tau_{k}\right)$. If $\tau_{k} \leq t<\tau_{k+1}$, then equation (1) coincides with equation (2), since $\mu(] \tau_{k}, \tau_{k+1}[, \mathbb{R})=0$ and thus the integral over the measure $\mu$ on the right hand side of (11) is equal to zero. Therefore we need to prove that $X_{1}\left(\tau_{k+1}\right)=X_{2}\left(\tau_{k+1}\right)$. Let $X^{*}(t)$ be a solution of equation (2) in the interval $\left[\tau_{k}, \tau_{k+1}\right.$ [ with the initial condition $X\left(\tau_{k}\right)$. This solution is unique, does not have discontinuities of the second kind, and thus is continuous almost surely at the point $t=\tau_{k+1}$, since the random variable $\tau_{k+1}$ does not depend on $X^{*}(t)$, has a continuous distribution, and the number of points of discontinuity 
of $X_{1}(t)$ is at most countable. A solution of equation (11) at the point $\tau_{k+1}$ can be expressed in terms of $X^{*}(t)$ in the interval $\left[\tau_{k}, \tau_{k+1}\right]$ as follows:

$$
\begin{aligned}
X_{1}\left(\tau_{k+1}\right) & =X^{*}\left(\tau_{k+1}-0\right)+q_{2}\left(\tau_{k+1}, X^{*}\left(\tau_{k+1}-0\right), \hat{\theta}_{k+1}\right) \\
& =X_{1}\left(\tau_{k+1}-0\right)+q_{2}\left(\tau_{k+1}, X_{1}\left(\tau_{k+1}-0\right), \hat{\theta}_{k+1}\right),
\end{aligned}
$$

where $\hat{\theta}_{k+1}$ is a point of $\mathbb{R}$ such that $\mu\left(\tau_{k+1}, \hat{\theta}_{k+1}\right)=1$. The equality

$$
X_{1}\left(\tau_{k+1}\right)=X_{2}\left(\tau_{k+1}\right)
$$

follows from $X_{1}\left(\tau_{k+1}-0\right)=X_{2}\left(\tau_{k+1}-0\right)$.

\section{The EXISTENCE OF A STRONG SOLUTiON}

In this section, we prove that the pathwise uniqueness of a solution and the existence of a weak solution of equation (2) imply the existence of a strong solution of the same equation.

Let $\mathfrak{X}=(X(t), W(t), \tilde{\nu}(t, d y))$ and $\mathfrak{X}^{\prime}=\left(X^{\prime}(t), W^{\prime}(t), \tilde{\nu}^{\prime}(t, d y)\right)$ be two solutions of equation (2) (generally speaking, these solutions are defined on different probability spaces) such that $X(0)=x$ and $X^{\prime}(0)=x$ almost surely, where $x \in \mathbb{R}$. Denote by $\mathcal{Z}$ the Fréchet space of real-valued functions on $[0, \infty)$ that are right continuous and have no discontinuities of the second kind. Let $\mathcal{Z}$ be equipped with the Skorokhod topology and let $\mathfrak{B}(\mathcal{Z})$ be the Borel $\sigma$-algebra in $\mathcal{Z}$. Further let $P\left(d w_{1} d w_{2} d w_{3}\right)$ and $P^{\prime}\left(d w_{1} d w_{2} d w_{3}\right)$ be the probability laws of the distributions of $\mathfrak{X}$ and $\mathfrak{X}^{\prime}$ in the space $(\mathcal{Z} \times \mathcal{Z} \times \mathcal{Z}, \mathfrak{B}(\mathcal{Z} \times \mathcal{Z} \times \mathcal{Z}))$, respectively.

Let $P_{w_{2} w_{3}}\left(d w_{1}\right)$ be a regular conditional probability $P\left(d w_{1} d w_{2} d w_{3}\right)$ given $w_{2}$ and $w_{3}$. This means that

(i) for all $w_{2}$ and $w_{3}, P_{w_{2} w_{3}}\left(d w_{1}\right)$ is a probability measure on $(\mathcal{Z}, \mathfrak{B}(\mathcal{Z}))$,

(ii) for all $B \in \mathfrak{B}(\mathcal{Z})$, the probabilities $P_{w_{2} w_{3}}(B)$ are $\mathfrak{B}(\mathcal{Z})$-measurable with respect to $w_{2}$ and $w_{3}$,

(iii) for all $B, B^{\prime}, B^{\prime \prime} \in \mathfrak{B}(\mathcal{Z})$,

$$
P\left(B \times B^{\prime} \times B^{\prime \prime}\right)=\int_{B^{\prime} \times B^{\prime \prime}} P_{w_{2} w_{3}}(B) R_{1}\left(d w_{2}\right) R_{2}\left(d w_{3}\right),
$$

where $R_{1}$ is a measure generated by $W(\cdot)$ and defined on $(\mathcal{Z}, \mathfrak{B}(\mathcal{Z})$ ) (that is, $R_{1}$ is a Wiener measure) and where $R_{2}$ is a measure generated by $\tilde{\nu}(\cdot, d y)$ and defined on $(\mathcal{Z}, \mathfrak{B}(\mathcal{Z}))$ (that is, $R_{2}$ is a centered Poisson measure).

Similarly we define $P_{w_{2} w_{3}}^{\prime}\left(d w_{1}\right)$ for $P^{\prime}\left(d w_{1} d w_{2} d w_{3}\right)$. Let $\mathfrak{B}_{t}(\mathcal{Z})$ be the Borel $\sigma$-algebra generated by $W(s)$ and $\tilde{\nu}(s, d y), s \leq t$. The $\sigma$-algebras $\mathfrak{B}_{t}(\mathcal{Z} \times \mathcal{Z}), \mathfrak{B}_{t}(\mathcal{Z} \times \mathcal{Z} \times \mathcal{Z})$, and $\mathfrak{B}_{t}(\mathcal{Z} \times \mathcal{Z} \times \mathcal{Z} \times \mathcal{Z})$ are defined similarly. Denote by $\mathfrak{B}_{\varepsilon}, \varepsilon \in(0,1]$, the $\sigma$-algebra of Borel subsets of $\mathbb{R}$ that belong to the set

$$
\left\{x: x \in \mathbb{R}, \varepsilon \leq|x| \leq \frac{1}{\varepsilon}\right\} .
$$

Finally let $\mathfrak{B}_{0}$ denote the union of $\sigma$-algebras $\mathfrak{B}_{\varepsilon}$ over all $\varepsilon \in(0,1]$.

We need the following auxiliary result.

Lemma 3.1. If $B \in \mathfrak{B}_{t}(\mathcal{Z})$, then $P_{w_{2} w_{3}}(B)$ (as well as $P_{w_{2} w_{3}}^{\prime}(B)$ ) is $\mathfrak{B}_{t}(\mathcal{Z})$-measurable with respect to $w_{2}$ and $w_{3}$.

Proof. Let $P_{w_{2} w_{3}}^{t}(\cdot)$ denote the regular conditional probability measure given $\mathfrak{B}_{t}(\mathcal{Z})$. This means that

(i) for all $w_{2}$ and $w_{3}, P_{w_{2} w_{3}}^{t}(\cdot)$ is a probability measure on $(\mathcal{Z}, \mathfrak{B}(\mathcal{Z}))$,

(ii) for all $B \in \mathfrak{B}(\mathcal{Z})$, the probability $P_{w_{2} w_{3}}^{t}(B)$ is $\mathfrak{B}_{t}(\mathcal{Z})$-measurable, 
(iii) for all $B \in \mathfrak{B}(\mathcal{Z})$ and $B^{\prime} \in \mathfrak{B}_{t}(\mathcal{Z})$,

$$
P\left(B \times B^{\prime} \times B^{\prime \prime}\right)=\int_{B^{\prime} \times B^{\prime \prime}} P_{w_{2} w_{3}}^{t}(B) R_{1}\left(d w_{2}\right) R_{2}\left(d w_{3}\right) .
$$

It is sufficient to prove that if $B \in \mathfrak{B}_{t}(\mathcal{Z})$, then $P_{w}(B)=P_{w}^{t}(B)$ almost surely. In its turn, this follows from

$$
\begin{aligned}
& \int_{\mathcal{Z} \times \mathcal{Z} \times \mathcal{Z}} F\left(w_{2}, w_{3}\right) I_{B}\left(w_{1}\right) P\left(d w_{1} d w_{2} d w_{3}\right) \\
& =\int_{\mathcal{Z} \times \mathcal{Z}} F\left(w_{2}, w_{3}\right) P_{w_{2} w_{3}}^{t}(B) R_{1}\left(d w_{2}\right) R_{2}\left(d w_{3}\right)
\end{aligned}
$$

if $F\left(w_{2}, w_{3}\right)$ is $\mathfrak{B}(\mathcal{Z})$-measurable and bounded.

Since $w_{2}$ and $w_{3}$ are processes with independent increments, we obtain from [7, pp. 19-21] that

$$
F\left(w_{2}, w_{3}\right)=c+\int_{0}^{\infty} \Phi_{1}(s) d w_{2}+\int_{0}^{\infty} \int_{\mathbb{R}} \Phi_{2}(s, y) d w_{3},
$$

where $\Phi_{1}(s)$ and $\Phi_{2}(s, y)$ are measurable and $\mathfrak{B}_{s}(\mathcal{Z})$-adapted processes for all $y \in \mathbb{R}$. Since $\left(W(t), \mathcal{F}_{t}\right)$ and $\left(\tilde{\nu}(t, A), \mathcal{F}_{t}\right), A \in \mathfrak{B}_{0}$, are martingales, we conclude that

$$
\left(w_{i}(t), \mathfrak{B}_{t}(\mathcal{Z} \times \mathcal{Z} \times \mathcal{Z}), P\left(d w_{1} d w_{2} d w_{3}\right)\right), \quad i=2,3,
$$

are also martingales.

Then

$$
\begin{aligned}
\int_{\mathcal{Z} \times \mathcal{Z} \times \mathcal{Z}} F\left(w_{2}, w_{3}\right) I_{B}\left(w_{1}\right) P\left(d w_{1} d w_{2} d w_{3}\right) \\
=c \int_{\mathcal{Z} \times \mathcal{Z} \times \mathcal{Z}} I_{B}\left(w_{1}\right) P\left(d w_{1} d w_{2} d w_{3}\right) \\
\quad+\int_{\mathcal{Z} \times \mathcal{Z} \times \mathcal{Z}}\left(\int_{t}^{\infty} \Phi_{1}(s) d w_{2}+\int_{t}^{\infty} \int_{\mathbb{R}} \Phi_{2}(s) d w_{3}\right) I_{B}\left(w_{1}\right) P\left(d w_{1} d w_{2} d w_{3}\right) \\
=\int_{\mathcal{Z} \times \mathcal{Z} \times \mathcal{Z}}\left(\int_{0}^{t} \Phi_{1}(s) d w_{2}+\int_{0}^{t} \int_{\mathbb{R}} \Phi_{2}(s) d w_{3}\right) I_{B}\left(w_{1}\right) P\left(d w_{1} d w_{2} d w_{3}\right)=: I_{1} .
\end{aligned}
$$

Since $\left(w_{i}(t), \mathfrak{B}_{t}(\mathcal{Z} \times \mathcal{Z} \times \mathcal{Z}), i=2,3\right.$, are martingales and $I_{B}\left(w_{1}\right)$ is $\mathfrak{B}_{t}(\mathcal{Z} \times \mathcal{Z} \times \mathcal{Z})$ measurable, we have

$$
\begin{aligned}
I_{1}= & c \int_{\mathcal{Z} \times \mathcal{Z}} P_{w_{2} w_{3}}^{t}(B) R_{1}\left(d w_{2}\right) R_{2}\left(d w_{3}\right) \\
& +\int_{\mathcal{Z} \times \mathcal{Z}}\left(\int_{0}^{t} \Phi_{1}(s) d w_{2}+\int_{0}^{t} \int_{\mathbb{R}} \Phi_{2}(s) d w_{3}\right) P_{w_{2} w_{3}}^{t}(B) R_{1}\left(d w_{2}\right) R_{2}\left(d w_{3}\right) \\
= & \int_{\mathcal{Z} \times \mathcal{Z}} F\left(w_{2}, w_{3}\right) P_{w_{2} w_{3}}^{t}(B) R_{1}\left(d w_{2}\right) R_{2}\left(d w_{3}\right),
\end{aligned}
$$

whence (4) follows. The lemma is proved.

Now we turn to the main result of this section.

Theorem 3.1. If a solution of equation (2) is pathwise unique, then a solution is unique in distribution.

Proof. We define a probability measure $Q\left(d w_{1} d w_{2} d w_{3} d w_{4}\right)$ on

$$
(\mathcal{Z} \times \mathcal{Z} \times \mathcal{Z} \times \mathcal{Z}, \mathfrak{B}(\mathcal{Z} \times \mathcal{Z} \times \mathcal{Z} \times \mathcal{Z}))
$$


as follows:

$$
Q\left(d w_{1} d w_{2} d w_{3} d w_{4}\right)=P_{w_{3} w_{4}}\left(d w_{1}\right) P_{w_{3} w_{4}}^{\prime}\left(d w_{2}\right) R_{1}\left(d w_{3}\right) R_{2}\left(d w_{4}\right) .
$$

Now we check that $\left[w_{i}(t), \mathfrak{B}_{t}(\mathcal{Z} \times \mathcal{Z} \times \mathcal{Z} \times \mathcal{Z})\right], i=3,4$, are martingales with respect to $Q$ and moreover $\left\langle w_{3}, w_{3}\right\rangle_{t}=t$ and $\left\langle w_{4}(\cdot, A), w_{4}(\cdot, A)\right\rangle_{t}=t \Pi(A)$ for all $A \in \mathfrak{B}_{0}$; that is, $w_{3}$ is a Wiener process and $w_{4}$ is a Poisson measure. Let $F_{i}, i=1, \ldots, 4$, be $\mathfrak{B}_{s}(\mathcal{Z})$-measurable bounded functions. Then

$$
\begin{aligned}
\int_{\mathcal{Z} \times \mathcal{Z} \times \mathcal{Z} \times \mathcal{Z}}\left[w_{3}(t)-w_{3}(s)\right] F_{1}\left(w_{1}\right) F_{2}\left(w_{2}\right) F_{3}\left(w_{3}\right) F_{4}\left(w_{4}\right) Q\left(d w_{1} d w_{2} d w_{3} d w_{4}\right) \\
=\int_{\mathcal{Z} \times \mathcal{Z}}\left[w_{3}(t)-w_{3}(s)\right]\left(\int_{\mathcal{Z}} F_{1}\left(w_{1}\right) P_{w_{3} w_{4}}\left(d w_{1}\right)\right)\left(\int_{\mathcal{Z}} F_{2}\left(w_{2}\right) P_{w_{3} w_{4}}^{\prime}\left(d w_{2}\right)\right) \\
\quad \times F_{4}\left(w_{4}\right) R_{1}\left(d w_{3}\right) R_{2}\left(d w_{4}\right) \\
\quad 0
\end{aligned}
$$

since $\int_{\mathcal{Z}} F_{1}\left(w_{1}\right) P_{w_{3} w_{4}}\left(d w_{1}\right)$ and $\int_{\mathcal{Z}} F_{2}\left(w_{2}\right) P_{w_{3} w_{4}}^{\prime}\left(d w_{2}\right)$ are $\mathfrak{B}_{s}(\mathcal{Z})$-measurable functions according to Lemma 3.1 .

Similarly we prove that

$$
\begin{gathered}
\int_{\mathcal{Z} \times \mathcal{Z} \times \mathcal{Z} \times \mathcal{Z}}\left[w_{4}(t, A)-w_{4}(s, A)\right] F_{1}\left(w_{1}\right) F_{2}\left(w_{2}\right) F_{3}\left(w_{3}\right) F_{4}\left(w_{4}\right) Q\left(d w_{1} d w_{2} d w_{3} d w_{4}\right)=0 \\
\int_{\mathcal{Z} \times \mathcal{Z} \times \mathcal{Z} \times \mathcal{Z}}\left\{\left[w_{3}(t)-w_{3}(s)\right]^{2}-(t-s)\right\} \\
\times F_{1}\left(w_{1}\right) F_{2}\left(w_{2}\right) F_{3}\left(w_{3}\right) F_{4}\left(w_{4}\right) Q\left(d w_{1} d w_{2} d w_{3} d w_{4}\right)=0 \\
\int_{\mathcal{Z} \times \mathcal{Z} \times \mathcal{Z} \times \mathcal{Z}}\left\{\left[w_{4}(t, A)-\right.\right. \\
\left.\left.\quad w_{4}(s, A)\right]^{2}-(t-s) \Pi(A)\right\} \\
\times F_{1}\left(w_{1}\right) F_{2}\left(w_{2}\right) F_{3}\left(w_{3}\right) F_{4}\left(w_{4}\right) Q\left(d w_{1} d w_{2} d w_{3} d w_{4}\right)=0
\end{gathered}
$$

Therefore $\left[w_{i}(t), \mathfrak{B}_{t}(\mathcal{Z} \times \mathcal{Z} \times \mathcal{Z} \times \mathcal{Z}), Q\right], i=3,4$, are the systems of martingales such that $\left\langle w_{3}, w_{3}\right\rangle_{t}=t$ and $\left\langle w_{4}(\cdot, A), w_{4}(\cdot, A)\right\rangle_{t}=t \Pi(A), A \in \mathfrak{B}_{0}$. Since $(X(t), W(t), \tilde{\nu}(t, d y))$ and $w_{1}, w_{3}, w_{4}$ are equivalent processes and the same holds for $\left(X^{\prime}(t), W^{\prime}(t), \tilde{\nu}^{\prime}(t, d y)\right)$ and $w_{2}, w_{3}, w_{4}$, we conclude that there are two solutions $\left(w_{1}, w_{3}, w_{4}\right)$ and $\left(w_{2}, w_{3}, w_{4}\right)$ defined on the same probability space

$$
\left(\mathcal{Z} \times \mathcal{Z} \times \mathcal{Z} \times \mathcal{Z}, \mathfrak{B}(\mathcal{Z} \times \mathcal{Z} \times \mathcal{Z} \times \mathcal{Z}), Q ; \mathfrak{B}_{t}(\mathcal{Z} \times \mathcal{Z} \times \mathcal{Z} \times \mathcal{Z})\right) .
$$

Since $w_{1}(0)=w_{2}(0)=x$ almost surely with respect to the measure $Q$, the pathwise uniqueness implies that $w_{1}(t)=w_{2}(t)$ almost surely with respect to the measure $Q$, whence we derive the uniqueness in distribution. Also,

$$
P_{w_{3} w_{4}} \times P_{w_{3} w_{4}}\left(w_{1}(t)=w_{2}(t)\right)=1,
$$

whence we obtain the existence of $F\left(w_{3}, w_{4}\right)$ such that $w_{1}=w_{2}=F\left(w_{3}, w_{4}\right)$. According to Lemma 3.1 the mapping $\left(w_{3}, w_{4}\right) \rightarrow F\left(w_{3}, w_{4}\right) \in \mathcal{Z}$ is $\mathfrak{B}_{t}(\mathcal{Z})$-measurable.

Corollary 3.1. If a solution of equation (2) is pathwise unique, and if there exists its solution $(X(t), W(t), \tilde{\nu}(t, d y))$ such that $X(0)=x \in \mathbb{R}$, then there exists a function $F\left(w_{1}, w_{2}\right): w_{1} \times w_{2} \in \mathcal{Z} \times \mathcal{Z} \rightarrow F\left(w_{1}, w_{2}\right) \in \mathcal{Z}$ such that $X(\cdot)=F(W(\cdot), \tilde{\nu}(\cdot, d y))$ almost surely. 


\section{MAin Result}

Below we prove the existence and uniqueness theorem for a strong solution of equation (11). We provide two proofs of this result. One of the proofs is based on Theorem 3.1 and its corollary, while the other one uses these results implicitly and is based on the classical theorem on the existence and uniqueness of a solution of an equation with nonLipschitz diffusion given in [4. Therefore the second proof is explicit, while the first one uses our Theorem 3.1, which is of an independent interest.

Theorem 4.1. Let the coefficients of equation (1) be such that

1) the functions $a(x), g(x)$, and $\int_{\mathbb{R}}\left|q_{1}(x, y)\right|^{2} \Pi(d y)$ are bounded;

2) the function $q_{2}(x, y)$ is continuous with respect to $x$ in the measure $m(d y)$;

3) $\lim _{x_{1} \rightarrow x_{2}} \int_{\mathbb{R}}\left|q_{1}\left(x_{1}, y\right)-q_{1}\left(x_{2}, y\right)\right|^{2} \Pi(d y)=0$;

4) there exists an increasing function $\rho(u)$ defined on $[0, \infty)$ and such that $\rho(0)=0$,

$$
\int_{0+} \rho^{-2}(u) d u=\infty
$$

and $|g(x)-g(y)| \leq \rho(|x-y|)$ for all $x, y \in \mathbb{R}$

5) $\left|a\left(x_{1}\right)-a\left(x_{2}\right)\right| \leq k\left|x_{1}-x_{2}\right|$ and $\int_{\mathbb{R}}\left|q_{1}\left(x_{1}, y\right)-q_{1}\left(x_{2}, y\right)\right| \Pi(d y) \leq k\left|x_{1}-x_{2}\right|$ for all $x_{1}, x_{2} \in \mathbb{R}$, where $k$ is a certain constant.

Then there exists a unique strong solution of equation (11).

Proof. Method I. By the assumptions of the theorem, equation (21) possesses a weak solution (see [2, p. 358]). According to Theorem [2.1, a solution of this equation is pathwise unique. Applying Corollary 3.1 of Theorem 3.1, we prove the existence of a strong solution of equation (2). Now we prove that a solution of equation (11) exists.

Denote by $\tau_{1}<\tau_{2}<\cdots<\cdots<\tau_{n}<\cdots$ the sequential stopping times that are the points of growth of the Poisson process $\mu((0, t], \mathbb{R})$. It is sufficient to construct a solution of equation (11) in every one of the intervals $\left[0, \tau_{1}\left[,\left[\tau_{1}, \tau_{2}\left[, \ldots,\left[\tau_{n}, \tau_{n+1}[, \ldots\right.\right.\right.\right.\right.$. Since

$$
\int_{\tau_{n}}^{t} \int_{\mathbb{R}} q_{2}(X(s), y) \mu(d s, d y)=0
$$

for $t \in\left[\tau_{n}, \tau_{n+1}[\right.$, equation (11) becomes of the form

$$
X(t)=X(\tau)+\int_{\tau}^{t} a(X(s)) d s+\int_{\tau}^{t} g(X(t)) d W(s)+\int_{\tau}^{t} \int_{\mathbb{R}} q_{1}(X(s), y) \tilde{\nu}(d s, d y)
$$

on every one of the above intervals, where $\tau$ is equal to $0, \tau_{1}, \tau_{2}, \ldots$ and so on. A solution of this equation exists if $X(\tau)$ is $\mathcal{F}_{t}$-measurable. We prove that this is the case, indeed. If $\tau=0$, then $X(0)$ is $\mathcal{F}_{0}$-measurable by the definition of the $\sigma$-algebra $\mathcal{F}_{t}$. Assume that $X\left(\tau_{n}\right)$ is $\mathcal{F}_{\tau_{n}}$-measurable. Then we show that $X\left(\tau_{n+1}\right)$ is $\mathcal{F}_{\tau_{n+1}}$-measurable. Let $X^{(n)}(t)$ be a solution of the equation

$$
X^{(n)}(t)=X\left(\tau_{n}\right)+\int_{\tau_{n}}^{t} a(X(s)) d s+\int_{\tau_{n}}^{t} g(X(t)) d W(s)+\int_{\tau_{n}}^{t} \int_{\mathbb{R}} q_{1}(X(s), y) \tilde{\nu}(d s, d y)
$$

for $t \geq \tau_{n}$. Put $X(t)=X^{(n)}(t)$ for $t<\tau_{n+1}$. Let $\theta_{n+1}$ be a point of $\mathbb{R}$ such that $\mu\left(\tau_{n+1}, \theta_{n+1}\right)=1$. Now we define $X\left(\tau_{n+1}\right)$ by the equality

$$
X\left(\tau_{n+1}\right)=X^{(n)}\left(\tau_{n+1}\right)+q_{2}\left(X^{(n)}\left(\tau_{n+1}\right), \theta_{n+1}\right) .
$$

Since $X^{(n)}(t)$ is $\mathcal{F}_{t}$-measurable, does not have discontinuities of the second kind, and is continuous with probability one at the point $\tau_{n+1}$, we conclude that $X^{(n)}\left(\tau_{n+1}\right)$ is $\mathcal{F}_{\tau_{n+1}}$-measurable. Similarly we prove that $\theta_{n+1}$ is $\mathcal{F}_{\tau_{n+1}}$-measurable. Thus both terms on the right hand side of (5) are $\mathcal{F}_{\tau_{n+1}}$-measurable, that is, $X\left(\tau_{n+1}\right)$ is $\mathcal{F}_{\tau_{n+1}}$-measurable. 
Therefore one can construct an $\mathcal{F}_{t}$-measurable process $X(t)$ step by step. This process is a solution of equation (11), indeed, since

$$
q_{2}\left(X^{(n)}\left(\tau_{n+1}\right), \theta_{n+1}\right)=\int_{\tau_{n}}^{\tau_{n+1}} q_{2}(X(s), y) \mu(d s, d y) .
$$

Applying the second statement of Theorem 2.1, we prove the existence and uniqueness of a strong solution of equation (11).

Method II. Consider the following differential equation:

$$
d X^{(m)}(t)=a\left(X^{(m)}(t)\right) d t+g\left(X^{(m)}(t)\right) d W(t)+\int_{\mathbb{R}} q_{1}^{(m)}\left(X^{(m)}(t), y\right) \tilde{\nu}(d t, d y),
$$

where

$$
q_{1}^{(m)}(x, y)=q_{1}(x, y) 1_{\left\{y \in \mathbb{R} \backslash S_{m}\right\}}
$$

and

$$
S_{m}=\{y:|y| \leq 1 / m\} .
$$

According to the definition of the function $q_{1}^{(m)}(x, y)$ and by the assumptions of the theorem, one can rewrite the integral

$$
\int_{\mathbb{R}} q_{1}^{(m)}\left(X^{(m)}(t), y\right) \tilde{\nu}(d t, d y)
$$

as follows:

$$
\begin{aligned}
\int_{\mathbb{R}} q_{1}^{(m)} & \left(X^{(m)}(t), y\right) \tilde{\nu}(d t, d y) \\
& =\int_{\mathbb{R}} q_{1}^{(m)}\left(X^{(m)}(t), y\right) \nu(d t, d y)-\int_{\mathbb{R}} q_{1}^{(m)}\left(X^{(m)}(t), y\right) \Pi(d y) d t .
\end{aligned}
$$

Then equation (6) becomes of the following form:

$$
\begin{aligned}
d X^{(m)}(t)=[ & \left.a\left(X^{(m)}(t)\right)-\int_{\mathbb{R}} q_{1}^{(m)}\left(X^{(m)}(t), y\right) \Pi(d y) d t\right] \\
& +g\left(X^{(m)}(t)\right) d W(t)+\int_{\mathbb{R}} q_{1}^{(m)}(X(t), y) \nu(d t, d y) .
\end{aligned}
$$

Now we consider the equation

$$
d X^{(m)}(t)=\left[a\left(X^{(m)}(t)\right)-\int_{\mathbb{R}} q_{1}^{(m)}\left(X^{(m)}(t), y\right) \Pi(d y) d t\right]+g\left(X^{(m)}(t)\right) d W(t) .
$$

The existence and uniqueness of a solution of this equation follows from [4, p. 173]. Following a reasoning similar to Method I, we prove the same result for a solution of equation (77) and, respectively, of equation (6). Now we check that the sequence of solutions $X^{(m)}(t)$ of equation (6) is a fundamental sequence.

We construct the functions $\varphi_{n}(x)$ as in the proof of Theorem 2.1. Consider the difference

$$
\begin{aligned}
X^{(l)}(t) & -X^{(m)}(t) \\
= & \int_{0}^{t}\left[a\left(X^{(l)}(s)\right)-a\left(X^{(m)}(s)\right)\right] d s+\int_{0}^{t}\left[g\left(X^{(l)}(s)\right)-g\left(X^{(m)}(s)\right)\right] d W(s) \\
& +\int_{0}^{t} \int_{\mathbb{R}}\left[q_{1}^{(l)}\left(X^{(l)}(s), y\right)-q_{1}^{(m)}\left(X^{(m)}(s), y\right)\right] \tilde{\nu}(d s, d y) .
\end{aligned}
$$


Applying Itô's formula to the function $\varphi_{n}(x)$ yields

$$
\begin{aligned}
\varphi_{n}\left(X^{(l)}(t)-X^{(m)}(t)\right) \\
=\int_{0}^{t} \varphi_{n}^{\prime}\left(X^{(l)}(s)-X^{(m)}(s)\right)\left[g\left(X^{(l)}(s)\right)-g\left(X^{(m)}(s)\right)\right] d W(s) \\
+\int_{0}^{t} \varphi_{n}^{\prime}\left(X^{(l)}(s)-X^{(m)}(s)\right)\left[a\left(X^{(l)}(s)\right)-a\left(X^{(m)}(s)\right)\right] d s \\
+\frac{1}{2} \int_{0}^{t} \varphi_{n}^{\prime \prime}\left(X^{(l)}(s)-X^{(m)}(s)\right)\left[g\left(X^{(l)}(s)\right)-g\left(X^{(m)}(s)\right)\right]^{2} d s \\
+\int_{0}^{t} \int_{\mathbb{R}}\left[\varphi_{n}\left(X^{(l)}(s)-X^{(m)}(s)+q_{1}^{(l)}\left(X^{(l)}(s), y\right)-q_{1}^{(m)}\left(X^{(m)}(s), y\right)\right)\right. \\
\quad-\varphi_{n}\left(X^{(l)}(s)-X^{(m)}(s)\right) \\
\quad-\left(q_{1}^{(l)}\left(X^{(l)}(s), y\right)-q_{1}^{(m)}\left(X^{(m)}(s), y\right)\right) \\
\left.\quad \times \varphi_{n}^{\prime}\left(X^{(l)}(s)-X^{(m)}(s)\right)\right] \Pi(d y) d s \\
+\int_{0}^{t} \int_{\mathbb{R}}\left[\varphi_{n}\left(X^{(l)}(s)-X^{(m)}(s)+q_{1}\left(X^{(l)}(s), y\right)-q_{1}\left(X^{(m)}(s), y\right)\right)\right. \\
\left.\quad-\varphi_{n}\left(X^{(l)}(s)-X^{(m)}(s)\right)\right] \tilde{\nu}(d s, d y) .
\end{aligned}
$$

Since the expectations of the first and the last terms on the right hand side of the latter equality are zero, we conclude that

$$
\begin{aligned}
& \mathrm{E}\left[\varphi_{n}\left(X^{(l)}(t)-X^{(m)}(t)\right)\right] \\
& =\mathrm{E}\left[\int_{0}^{t} \varphi_{n}^{\prime}\left(X^{(l)}(s)-X^{(m)}(s)\right)\left\{a\left(X^{(l)}(s)\right)-a\left(X^{(m)}(s)\right)\right\} d s\right] \\
& +\frac{1}{2} \mathrm{E}\left[\int_{0}^{t} \varphi_{n}^{\prime \prime}\left(X^{(l)}(s)-X^{(m)}(s)\right)\left\{g\left(X^{(l)}(s)\right)-g\left(X^{(m)}(s)\right)\right\}^{2} d s\right] \\
& +\mathrm{E}\left[\int _ { 0 } ^ { t } \int _ { \mathbb { R } } \left\{\varphi_{n}\left(X^{(l)}(s)-X^{(m)}(s)+q_{1}^{(l)}\left(X^{(l)}(s), y\right)-q_{1}^{(m)}\left(X^{(m)}(s), y\right)\right)\right.\right. \\
& \quad-\varphi_{n}\left(X^{(l)}(s)-X^{(m)}(s)\right) \\
& \quad-\left(q_{1}^{(l)}\left(X^{(l)}(s), y\right)-q_{1}^{(m)}\left(X^{(m)}(s), y\right)\right) \\
& \left.\left.\quad \times \varphi_{n}^{\prime}\left(X^{(l)}(s)-X^{(m)}(s)\right)\right\} \Pi(d y) d s\right] \\
& =I_{1}+I_{2}+I_{3},
\end{aligned}
$$

where

$$
\begin{aligned}
& \left|I_{1}\right| \leq \int_{0}^{t} \mathrm{E}\left[\left|a\left(X^{(l)}(s)\right)-a\left(X^{(m)}(s)\right)\right|\right] d s \leq \int_{0}^{t} k\left[\mathrm{E}\left(\left|X^{(l)}(s)-X^{(m)}(s)\right|\right)\right] d s, \\
& \left|I_{2}\right| \leq \frac{1}{2} \int_{0}^{t} \mathrm{E}\left[\frac{2}{n} \rho^{-2}\left(\left|X^{(l)}(s)-X^{(m)}(s)\right|\right) \rho^{2}\left(\left|X^{(l)}(s)-X^{(m)}(s)\right|\right)\right] d s \leq \frac{t}{n} \rightarrow 0
\end{aligned}
$$

as $n \rightarrow \infty$. 
Consider the term $I_{3}$. We apply the Lagrange formula to $\varphi_{n} \in C^{2}(\mathbb{R})$ :

$$
\begin{aligned}
\varphi_{n}\left(X^{(l)}(s)-X^{(m)}(s)+q_{1}^{(l)}\left(X^{(l)}(s), y\right)-q_{1}^{(m)}\left(X^{(m)}(s), y\right)\right) \\
-\varphi_{n}\left(X^{(l)}(s)-X^{(m)}(s)\right) \\
=\varphi_{n}^{\prime}(\theta)\left(q_{1}^{(l)}\left(X^{(l)}(s), y\right)-q_{1}^{(m)}\left(X^{(m)}(s), y\right)\right),
\end{aligned}
$$

where $\theta=\theta(n, y)$ is some intermediate point. Since $\left|\varphi_{n}^{\prime}(x)\right| \leq 1$, we obtain

$$
\begin{aligned}
\left|I_{3}\right| \leq \mathrm{E} \int_{0}^{t} \int_{\mathbb{R}} \mid \varphi_{n}^{\prime}(\theta)\left(q_{1}^{(l)}\left(X^{(l)}(s), y\right)-q_{1}^{(m)}\left(X^{(m)}(s), y\right)\right) \\
\quad-\varphi_{n}^{\prime}\left(X^{(l)}(s)-X^{(m)}(s)\right) \\
\quad \times\left(q_{1}^{(l)}\left(X^{(l)}(s), y\right)-q_{1}^{(m)}\left(X^{(m)}(s), y\right)\right) \mid \Pi(d y) d s \\
\leq 2 \mathrm{E} \int_{0}^{t} \int_{\mathbb{R}}\left|q_{1}^{(l)}\left(X^{(l)}(s), y\right)-q_{1}^{(m)}\left(X^{(m)}(s), y\right)\right| \Pi(d y) d s \\
\leq 2 \mathrm{E} \int_{0}^{t} \int_{\mathbb{R}}\left|q_{1}^{(l)}\left(X^{(l)}(s), y\right)-q_{1}^{(l)}\left(X^{(m)}(s), y\right)\right| \Pi(d y) d s \\
\quad+2 \mathrm{E} \int_{0}^{t} \int_{\mathbb{R}}\left|q_{1}^{(l)}\left(X^{(m)}(s), y\right)-q_{1}^{(m)}\left(X^{(m)}(s), y\right)\right| \Pi(d y) d s \\
=I_{4} .
\end{aligned}
$$

For definiteness, let $l>m$. Then

$$
\begin{aligned}
I_{4} \leq & 2 \mathrm{E} \int_{0}^{t} \int_{\mathbb{R}}\left|q_{1}\left(X^{(l)}(s), y\right)-q_{1}\left(X^{(m)}(s), y\right)\right| 1_{\{|y|>1 / l\}} \Pi(d y) d s \\
& +\int_{0}^{t} \int_{\mathbb{R}}\left|q_{1}\left(X^{(m)}(s), y\right)\right| 1_{\{1 / l<|y| \leq 1 / m\}} \Pi(d y) d s \\
\leq & 2 \int_{0}^{t} k\left[\mathrm{E}\left|X^{(l)}(s)-X^{(m)}(s)\right|\right] d s+2 \mathrm{E} \int_{0}^{t} \int_{S_{m} \backslash S_{l}}\left|q_{1}\left(X^{(m)}(s), y\right)\right| \Pi(d y) d s \\
\leq & 2 \int_{0}^{t} k\left[\mathrm{E}\left|X^{(l)}(s)-X^{(m)}(s)\right|\right] d s \\
& +2 \mathrm{E} \int_{0}^{t}\left(\int_{S_{m} \backslash S_{l}}\left|q_{1}\left(X^{(m)}(s), y\right)\right|^{2} \Pi(d y)\right)^{1 / 2}\left(\Pi\left(S_{m} \backslash S_{l}\right)\right)^{1 / 2} d s \\
\leq & 2 \int_{0}^{t} k\left[\mathrm{E}\left|X^{(l)}(s)-X^{(m)}(s)\right|\right] d s+I_{5} .
\end{aligned}
$$

Passing to the limit as $n \rightarrow \infty$ we get

$$
\mathrm{E}\left(\left|X^{(l)}(t)-X^{(m)}(t)\right|\right) \leq \int_{0}^{t} k\left[\mathrm{E}\left(\left|X^{(l)}(s)-X^{(m)}(s)\right|\right)\right] d s+I_{5} .
$$

This implies that $\mathrm{E}\left(\left|X^{(l)}(s)-X^{(m)}(s)\right|\right) \leq I_{5} e^{k t}$. Since $I_{5} \rightarrow 0$ as $m \rightarrow \infty$,

$$
\mathrm{E}\left(\left|X^{(l)}(s)-X^{(m)}(s)\right|\right) \rightarrow 0, \quad m, n \rightarrow \infty,
$$

whence we conclude that $\left\{X^{(m)}(t)\right\}$ is a fundamental sequence. Thus $\left\{X^{(m)}(t)\right\}$ converges in the mean square sense to some limit $X(t)$. Now we show that $X(t)$ is a solution 
of equation (2). Indeed,

$$
\mathrm{E}\left|\int_{0}^{t}\left[a(X(s))-a\left(X^{(m)}(s)\right)\right] d s\right| \leq \mathrm{E} \int_{0}^{t}\left|a(X(s))-a\left(X^{(m)}(s)\right)\right| d s \rightarrow 0
$$

as $m \rightarrow \infty$, since the function $a(x)$ is continuous and bounded and $X^{(m)}(s) \rightarrow X(s)$ in probability. Further,

$$
\begin{aligned}
\mathrm{E} \mid \int_{0}^{t} & {\left[g(X(s))-g\left(X^{(m)}(s)\right)\right] d W(s) \mid } \\
& \leq\left(\mathrm{E}\left|\int_{0}^{t}\left[g(X(s))-g\left(X^{(m)}(s)\right)\right] d W(s)\right|^{2}\right)^{1 / 2} \\
& =\left(\mathrm{E} \int_{0}^{t}\left[g(X(s))-g\left(X^{(m)}(s)\right)\right]^{2} d s\right)^{1 / 2} \rightarrow 0, \quad m \rightarrow \infty
\end{aligned}
$$

since the function $g(x)$ is continuous and bounded and $X^{(m)}(s)$ converges to $X(s)$ in probability. Finally,

$$
\begin{aligned}
& \mathrm{E}\left|\int_{0}^{t} \int_{\mathbb{R}}\left[q_{1}(X(s), y)-q_{1}^{(m)}\left(X^{(m)}(s), y\right)\right] \tilde{\nu}(d s, d y)\right| \\
& \quad \leq\left(\mathrm{E}\left|\int_{0}^{t} \int_{\mathbb{R}}\left[q_{1}(X(s), y)-q_{1}^{(m)}\left(X^{(m)}(s), y\right)\right] \tilde{\nu}(d s, d y)\right|^{2}\right)^{1 / 2} \\
& \quad=\left(\mathrm{E} \int_{0}^{t} \int_{\mathbb{R}}\left|q_{1}(X(s), y)-q_{1}^{(m)}\left(X^{(m)}(s), y\right)\right|^{2} \Pi(d y) d s\right)^{1 / 2} \\
& \leq\left(\mathrm{E} \int_{0}^{t} \int_{\mathbb{R}}\left|q_{1}(X(s), y)-q_{1}\left(X^{(m)}(s), y\right)\right|^{2} 1_{\{|y|>1 / m\}} \Pi(d y) d s\right. \\
& \left.\quad+\mathrm{E} \int_{0}^{t} \int_{\mathbb{R}}\left|q_{1}(X(s), y)\right|^{2} 1_{\{|y| \leq 1 / m\}} \Pi(d y) d s\right)^{1 / 2} \rightarrow 0
\end{aligned}
$$

as $m \rightarrow \infty$. The latter result holds, since $\int_{\mathbb{R}}\left|q_{1}(x, y)\right|^{2} \Pi(d y)$ is bounded and one can follow the same reasoning as above by using the assumption on the continuity.

Hence $X(t)$ is a solution of equation (2), indeed. As we have already proved, a unique solution of equation (6) exists and the same is true for equation (2). The rest of the proof of the existence and uniqueness of a strong solution of equation (11) is the same as that in Method I.

Remark 4.1. One can generalize Theorems 2.1 and 4.1 to the case where the coefficients of a stochastic differential equation grow linearly.

\section{Concluding Remarks}

Studies of stochastic differential diffusion type equations with non-Lipschitz coefficients are of special interest for modeling interest rates of nonrisky assets, since one of the principal goals of financial institutions and insurance companies is to manage risks caused by fluctuations of interest rates [8]. However, the real markets are functioning in such a way that interest rates jump at certain time instances. Therefore the questions considered in this paper concerning the existence and uniqueness of a solution of equation (11) allow one to model the interest rates of such financial markets and thus may have not only theoretical but also some applied value. 


\section{BIBLIOGRAPHY}

1. I. I. Gikhman and A. V. Skorokhod, Stochastic Differential Equations, Naukova Dumka, Kiev, 1968; English transl., Springer-Verlag, New York-Heidelberg, 1972. MR0263172 (41:7777) MR0346904 (49:11625)

2. I. I. Gikhman and A. V. Skorokhod, Stochastic Differential Equations and their Applications, Naukova Dumka, Kiev, 1982. (Russian) MR678374 (84j:60003)

3. D. W. Stroock and S. R. S. Varadhan, Multidimensional Diffusion Processes, Springer-Verlag, Berlin, 1979. MR532498 (81f:60108)

4. N. Ikeda and S. Watanabe, Stochastic Differential Equations and Diffusion Processes, second edition, North-Holland, Amsterdam, 1989. MR 1011252 (90m:60069)

5. T. Yamada and S. Watanabe, On the uniqueness of solutions of stochastic differential equations, J. Math. Kyoto Univ. 11 (1971), no. 1, 155-167. MR0278420 (43:4150)

6. G. L. Kulinich, On existence and uniqueness of a solution of a stochastic differential equation with martingale differential, Teor. Veroyatnost. Primenen. XIX (1974), no. 1, 169-173; English transl. in Theory Probab. Appl. 19 (1974-1975), no. 1, 168-171. MR0345209 (49:9948)

7. L. I. Gal'chuk, The structure of some martingales, Proceedings of the School Seminar on the Theory of Stochastic Processes, vol. 1, Vilnius, 1975, pp. 9-33. (Russian)

8. V. Zubchenko, Long-term returns in stochastic interest rate models, Theory Stoch. Process. $\mathbf{1 3}$ (2007), no. 4, 247-261. MR2482264 (2001h:60188)

Department of Probability Theory and Mathematical Statistics, Faculty for Mechanics and Mathematics, Kyiv National Taras Shevchenko University, Academician Glushkov AvEnUe, 2, Kiev 03127, Ukraine

E-mail address: v_zubchenko@ukr.net

Department of Probability Theory and Mathematical Statistics, Faculty for Mechanics and Mathematics, Kyiv National Taras Shevchenko University, Academician Glushkov AvENUE, 2, Kiev 03127, Ukraine

E-mail address: myus@univ.kiev.ua

Received 25/FEB/2009

Translated by N. N. SEMENOV 